\title{
Formations expérimentales pour les universités du Sud
}

La CIRUISEF et I'Université Paris-Est Créteil, avec le soutien de l'Université Paris-Sud 11 et de l'Université de Provence, ont organisé du 9 au 20 janvier 2012 un stage de formation expérimentale à l'attention des maîtres de conférence et des ingénieurs de physique des universités francophones des pays du Sud, mettant en œuvre une « armoire de la physique ». 17 participants, provenant de 9 pays, ont été accueillis, et sont repartis avec un matériel permettant de démarrer immédiatement des expériences chez eux.

pas qu'on
pouvait faire
et apprendre
en si peu
de temps.,
Le réseau CIRUISEF (encadré 1) rassemble 126 doyens de facultés de sciences des universités francophones et 57 responsables affiliés. Depuis plusieurs années, il a examiné avec les partenaires du Sud (Afrique principalement) et du Nord (France, Belgique, Canada) les divers niveaux de formations universitaires, d'organisation de la recherche, puis de formation de réseaux et échanges internationaux Nord-Sud, mais aussi, autant que possible, Sud-Sud.

Au-delà des spécificités des niveaux, des contenus des programmes et des pays, une même insuffisance est constatée : la faiblesse des formations expérimentales universitaires, qui ne fait que prolonger celle des années antérieures à partir de l'enseignement primaire jusqu'au secondaire (où les pratiques de type "main à la pâte " sont généralement inconnues). Ce déficit vient en partie de l'insuffisance d'équipement expérimental ou de locaux dédiés... mais aussi, et peut-être surtout, le plus souvent, à l'absence de formation des enseignants qui rebondit en cascade sur les élèves de tous les niveaux. Or, les études scientifiques expérimentales (physique, chimie, biologie, géologie) nécessitent, pour être comprises et assimilées par les étudiants, une relation personnelle avec la matière : observation, conception, utilisation, adaptation, interprétation. La créativité et l'aptitude à développer des applications nécessitent ce contact.

Consciente de ce problème, la CIRUISEF, que préside énergiquement Madame Évelyne Garnier-Zarli, doyenne de l'Université Paris-Est Créteil, avait mis en place un programme de "valises de chimie " contenant un kit de matériel de base permettant une initiation à l'expérimentation ; ces valises ont été distribuées à un certain nombre d'universités.

À la suite de cette première réalisation, plusieurs enseignants et ingénieurs de recherche ont envisagé la mise en œuvre d'une " armoire de la physique ", rassemblant de même un matériel de base de physique et proposant une série de travaux pratiques (encadré 2) pouvant être prolongés par la suite par des projets plus originaux. L'ambition était de faire financer un ensemble de ces " armoires ", à mettre en place dans quelques universités du Sud, avec l'ambition que celles-ci puissent les dupliquer. L'ensemble ainsi réalisé a été transporté et testé avec succès pour la première fois à l'occasion d'un congrès Nord-Sud, organisé par la Société Française de Physique à Oujda en $2007^{(1)}$. Les délais de financement et des contacts difficiles avec une société de matériel pédagogique censée nous assister, ont freiné sans l'interrompre la suite de ce projet.

Ainsi, nous avons pu, sur une quinzaine de jours en janvier 2012, accueillir (avec le soutien des ambassades) dix-sept jeunes enseignants et ingénieurs universitaires représentant neuf pays du Sud ${ }^{(2)}$, à une introduction aux pratiques expérimentales à partir d'un matériel de base de cette armoire et de compléments pouvant être facilement obtenus sur place. Il s'est agi essentiellement de travail "en paillasse ", où nous avons pu constater à la fois la curiosité scientifique de tous ces enseignants et le besoin de cette initiation, incluant les gestes et pratiques de base de l'expérimentation scientifique et technique ${ }^{(3)}$.

\section{La CIRUISEF}

Créée en 1988, la CIRUISEF (www.ciruisef.com), Conférence internationale des responsables des universités et institutions à dominante scientifique et technique d'expression française, est l'un des principaux réseaux institutionnels de l'Agence universitaire de la Francophonie (AUF). Elle a pour objectif de rassembler les institutions universitaires francophones du Nord et du Sud, dans le domaine des sciences exactes, naturelles et technologiques.

L'essentiel de ses activités porte sur:

- la conception, l'élaboration, la diffusion d'outils pédagogiques adaptés à l'enseignement des sciences en français ;

- I'organisation de colloques annuels, relatifs à des thématiques scientifiques ou pédagogiques larges, intéressant et rassemblant des enseignants-chercheurs et étudiants du Nord et du Sud. 

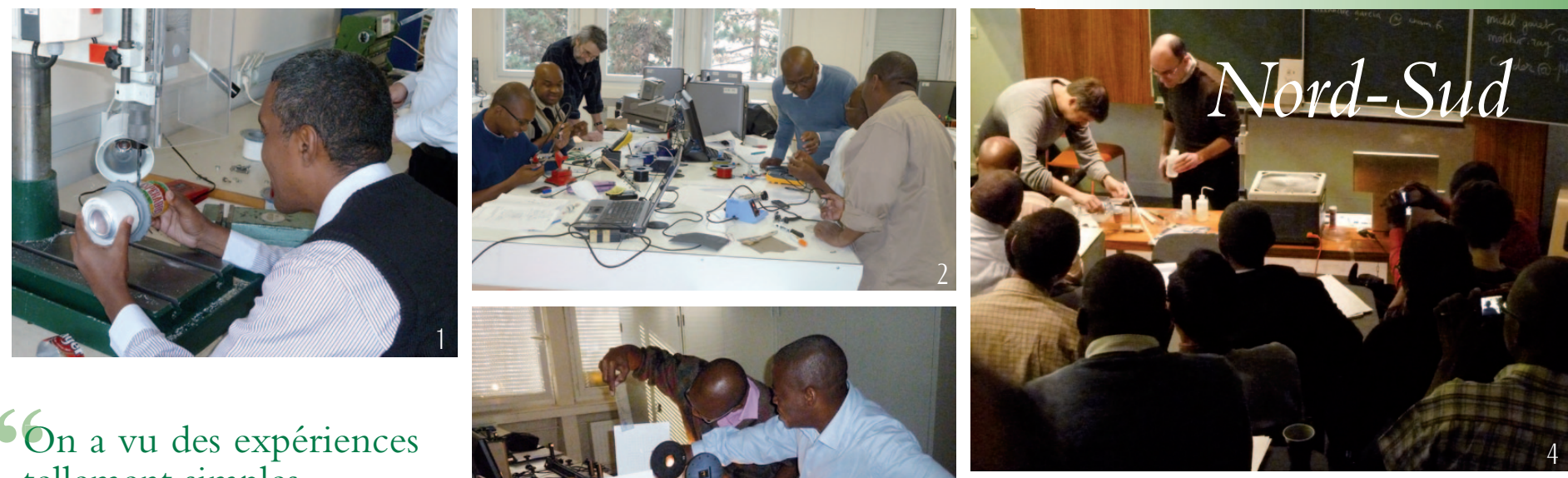

On a vu des expériences tellement simples, qui font comprendre des phénomènes si compliqués.

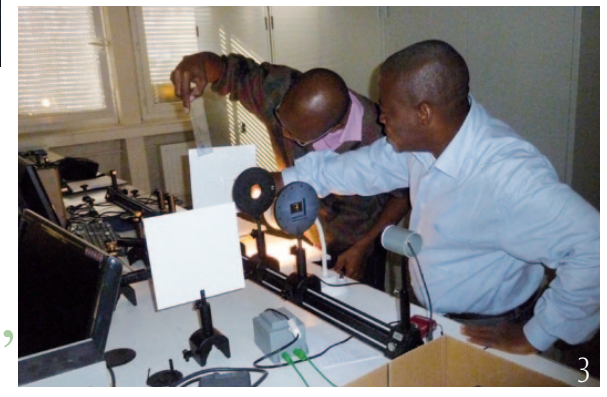

1. Confection d'un calorimètre « maison ».

2. Montage d'un régulateur de températures (électronique).

3. Focométrie au banc d'optique.

4. Démonstrations sur la tension superficielle (conférence),

Ces séances, effectuées dans les salles de travaux pratiques des universités de Créteil et d'Orsay avec, de plus, la participation de collègues de l'Université de Provence (qui avaient déjà eu une expérience suivie de collaboration sur les TP avec des collègues malgaches), se sont conclues en donnant aux participants en fin de séjour un matériel de base (l'armoire !) leur permettant de reproduire, adapter et dupliquer ces expérimentations chez eux.

Nous avons aussi pensé important d'encourager nos partenaires africains à utiliser un matériel simple pour des expérimentations originales ne nécessitant pas un équipement important et susceptibles, si possible, de s'inscrire dans le cadre de thèmes d'intérêt local (énergie, eau, sols...). Pour cela, nous avons organisé à l'ESPCI une série de six conférences expérimentales données par des physiciens qui se sont impliqués dans ce type d'activité, en prolongement de leur enseignement universitaire plus classique. La présentation d'Yves Couder sur l'enseignement expérimental de licence à
Paris 7 en a défini la nature et le style ; elle a été complétée par des présentations de Bernard Valeur et Alexandre Garcia (lumière et sons), José Bico et Benoît Roman (la mécanique des solides), Christophe Clanet (la mécanique des balles), Marc Rabaud (les ondes de surface), Étienne Guyon et Patrice Jenffer (suspensions et poreux).

Naturellement, cette expérience ne prendra son sens que si ce premier essai se prolonge et se diversifie autour de chaque projet vu comme un prototype, et que si des soutiens financiers (AUF, ministère des Affaires étrangères, entreprises...) sont obtenus pour la consolider. Un suivi personnalisé des collègues stagiaires est commencé.

Pour continuer et développer cette première expérience, l'aide de nouveaux collègues physiciens souhaitant s'impliquer dans ce type d'action de coopération sera bienvenue.

Michel Gouet (gouet@univ-paris12.fr) et Étienne Guyon, au nom du comité " Armoire de la physique "
(1) Congrès Nord-Sud de Physique, 9-13 avril 2007, Université Mohamed $1^{\text {er }}$, Oujda,

Maroc (http://arxiv. org/pdf/0711.0845.pdf) Voir aussi Reflets de la physique $\mathrm{n}^{\circ} \mathbf{4}$ (mai 2007), p. 2 .

(2) Burkina Faso, Cameroun, Congo Brazzaville, Côte d'Ivoire, Liban, Madagascar, Mali, Sénégal, Togo.

(3) Un compte rendu détaillé et illustré peut être trouvé sur le site www.ciruisef.com rubrique Actions/ Armoire TP Physique.

\section{Quelques participants donnent leur opinion sur le stage}

${ }^{6}$ On a eu le plaisir

de fabriquer, même en se brûlant les doigts avec le fer à souder.

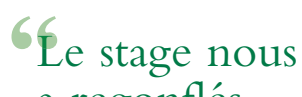

a regonflés.,
G $G$ e me suis rendu compte que nous pouvons déjà beaucoup avancer avec le matériel que nous avons ou que nous pouvons fabriquer. Nous avons vu qu'on peut facilement lier des cours théoriques à des expériences qui ne demandent pas beaucoup de moyens.,

- Expériences d'optique.

- Expériences de thermique (calorimétrie, fusion de la glace, régulation de température, enregistrement).

- Expériences de mécanique (addition des forces en vecteurs, pendules, ressorts, inertie, chute de bille parabolique, chute avec vitesse initiale).

- Expériences d'électricité (courant continu, courant alternatif, utilisation de multimètres, mesures sur des résistances linéaires, circuits résistance + inductance et résistance + capacité, décharge d'un condensateur).

- Expériences de physique des fluides (dynamique des fluides, tension superficielle, mesure de la viscosité par chute de billes, phénomènes d'interface).

- Réalisation de montages électriques, thermiques, mécaniques, optiques...

Certains de ces travaux pratiques sont décrits dans un document téléchargeable sur le site www.ciruisef.com, rubrique Actions/Armoire TP Physique. 